\title{
Transient Neonatal Hypothyroxinemia and the Auditory Brainstem Evoked Response
}

\author{
DAVID KOHELET, ELIANA ARBEL, MICHAEL GOLDBERG, AND AHARON ARLAZZOROFF \\ Departments of Neonatology [D.K., E.A., M.G.] and Neurology [A.A.], Assaf Harofeh Medical Center, Zerifin, \\ and the Sackler School of Medicine, Tel Aviv University, Tel Aviv, Israel
}

\begin{abstract}
Ten premature infants with low serum thyroxine levels (less than $84 \mathrm{nmol} / \mathrm{L}$ ) were compared with 10 biochemically euthyroid infants to determine whether hypothyroxinemia in such infants can lead to alterations in the auditory pathway. The auditory pathway was studied between 6 and $11 \mathrm{wk}$ of life by analyzing brainstem evoked potentials elicited by a $10 / \mathrm{s}, 75 \mathrm{~dB}$ above normal adult hearing level click stimulus presented at the infant's ears. Peak latencies of components I, III, and $\mathrm{V}$ and interpeak latencies I-III, III-V, and I-V did not yield statistically significant differences between groups. The present study indicates that untreated neonatal hypothyroxinemia does not lead to abnormalities of auditory brainstem evoked response. (Pediatr Res 32: 530-531, 1992)
\end{abstract}

Abbreviations

$T_{4}$, thyroxine

dB nHL, decibels above normal adult hearing level

The thyroid gland plays a significant role in the maturation of the brain. Untreated hypothyroidism may be associated with irreversible damage to the CNS. Temporary hypothyroxinemia as distinct from congenital hypothyroidism is a well-known finding in the small, premature infant. In such cases, the low $\mathrm{T}_{4}$ levels usually revert to normal after a variable period of time (1). The significance of this observation is not clear, and an important issue raised by the identification of these preterm infants is whether or not replacement hormonal treatment is required (2). Evaluation of auditory brainstem potentials in premature infants with such hypothyroxinemia may well provide information regarding any deleterious effect on the development of the auditory brainstem pathway resultant from low hormone levels. Our study investigated this aspect.

\section{SUBJECTS AND METHODS}

A study group of 10 preterm infants with untreated transient hypothyroxinemia (mean $\mathrm{T}_{4}: 49.9 \pm 0.03 \mathrm{nmol} / \mathrm{L}$; mean TSH: $13.2 \pm 2.0 \mathrm{mU} / \mathrm{L}$ ) was compared with a matched control group of 10 preterm infants with $\mathrm{T}_{4}$ levels greater than $84 \mathrm{nmol} / \mathrm{L}$. All infants were admitted at birth to the Neonatal Intensive Care Unit at the Assaf Harofeh Medical Center, Zerifin, Israel.

All infants were screened for congenital hypothyroidism at the age of 3-10 d with a heel-stick specimen of blood collected onto an approved filter paper form and analyzed for $T_{4}$ levels by CoatA-Count Neonatal $\mathrm{T}_{4}$ Radioimmunoassay (Diagnostic Products Corporation, Los Angeles, CA). If the $\mathrm{T}_{4}$ level was below a

Received March 10, 1992; accepted July 9, 1992.

Correspondence: Dr. D. Kohelet, Department of Neonatology, Assaf Harofeh Medical Center, 70300 Zerifin, Israel. certain cutoff point established by the laboratory, a TSH assay was done on the same specimen using the Coat-A-Count Neonatal TSH Radioimmunoassay (Diagnostic Products Corporation). Hypothyroxinemia was defined as a $\mathrm{T}_{4}$ value less than 84 $\mathrm{nmol} / \mathrm{L}$ with a concomitant TSH value less than $20 \mathrm{mU} / \mathrm{L}$ on two occasions. All infants with initially low screen $\mathrm{T}_{4}$ levels had repeat blood samples for $\mathrm{T}_{4}$ and $\mathrm{TSH}$ until $\mathrm{T}_{4}$ levels became normal. The control group, which screened euthyroid, was randomly chosen and closely matched with the study group cared for in the unit during the same period. The matching factors included birth weight, gestational age, and conceptional age at which time the brainstem auditory evoked response was elicited. Gestational age was assessed by maternal dates with confirmation by the method of Ballard et al. (3). Conceptional age was defined as gestational age plus postnatal age at the time of the auditory evoked response. None of the infants in either group evidenced severe perinatal asphyxia (Apgar score less than 6 at $5 \mathrm{~min}$ of age), hyperbilirubinemia requiring exchange transfusion, signs of maternal or fetal infection, clinical features of brain damage, or a family history of deafness. All infants were seen in the followup clinic and underwent full neurologic examination at 4, 6, 9, 12 , and $18 \mathrm{mo}$ of age. None of the infants had evidence of neurodevelopmental delay. Selected clinical data are given in Table 1.

All infants admitted to the Neonatal Intensive Care Unit undergo routine auditory evoked response examination before discharge or shortly thereafter (between 6 and 11 wk of age). The examination was performed on a Microshev neuroscope (Eilon Shvut, Israel) and carried out when the infants' health and weight permitted safe transport to the neurology department where the neuroscope was situated. All infants were tested by the same operator about an hour after feeding. Each infant was asleep during the examination. The measurements were carried out in both groups at similar conceptional ages. Only three infants in the study group had abnormal $\mathrm{T}_{4}$ levels when the examination was made. All infants had initial brainstem auditory evoked response assessment via a click stimulus presented through supraaural earphones taped over the ears at a repetition rate of $10 /$ $\mathrm{s}$. The device was set to $0.2 \mathrm{mV}$ sensitivity and $10 \mathrm{~ms}$ analysis

Table 1. Clinical data on study and control group

\begin{tabular}{lcc}
\hline & Study group & Control group \\
\hline Birth weight (g) & $1232 \pm 360$ & $1251 \pm 249$ \\
Gestational age (wk) & $30.9 \pm 3.9$ & $30.9 \pm 2.4$ \\
Conceptional age (wk) & $39.2 \pm 2.3$ & $41.7 \pm 5.3$ \\
Gender (m:f) & $4: 6$ & $3: 7$ \\
Mechanical ventilation (no.) & 3 & 4 \\
Intraventricular hemorrhage (no.) & 2 & 1 \\
Gentamicin treated (no.) & 9 & 9 \\
Aminophyllin treated (no.) & 10 & 10 \\
Patent ductus arteriosus (no.) & 1 & 2 \\
Air leaks (no.) & 0 & 0 \\
Peak bilirubin value ( $\mu$ mol/L) & $186 \pm 43$ & $167 \pm 50$
\end{tabular}


time. Eleven hundred recordings at 30 and $60 \mathrm{~dB}$ nHL (above the normal thresholds to click stimuli established in individuals with normal audiograms) for each ear were averaged. Responses were recorded with electrodes at the vertex, referenced to the earlobe. The forehead was grounded. The initial 30- and 60-dB nHL clicks were selected according to recommendations made in an earlier study (4). All infants yielded normal auditory evoked responses at these click intensities. The examination was then carried out on each infant at a click intensity of $75 \mathrm{~dB} n \mathrm{~nL}$ and a repetition rate of $10 / \mathrm{s}$ (100 ms between stimuli). Components I, III, and V were remarkably consistent in both form and amplitude. The latencies were reproducible and easily defined. Components were defined at their peak. Six parameters, namely peak latency of components I, III and V and interpeak latency of intervals I-III, III-V, and I-V, were measured in $\mathrm{ms}$ and recorded for each ear. Differences between means were studied using unpaired $t$ tests. A level of significance was set at $p<0.01$ to minimize statistical error due to multiple $t$ test. The Fisher exact test was used to compare proportions among groups.

\section{RESULTS}

Tables 2 and 3 indicate the mean peak latencies of waves I, III, and V and interpeak latencies I-III, III-V, and I-V, respectively, ear by ear in each group at a click intensity of $75 \mathrm{~dB}$ nHL. The values for each of the six parameters were similar and any observed differences did not reach statistical significance. Al-

Table 2. Comparison of peak latency values between groups at click intensity of $75 \mathrm{~dB} n \mathrm{HL}$

\begin{tabular}{rccccc}
\hline & \multicolumn{4}{c}{ Peak latency value (ms) } \\
\cline { 2 - 3 } Wave & \multicolumn{2}{c}{ Study group } & & \multicolumn{2}{c}{ Control group } \\
\cline { 2 - 3 } \cline { 5 - 6 } I & $1.92 \pm 0.31$ & $1.85 \pm 0.28$ & & $1.84 \pm 0.13$ & $1.86 \pm 0.12$ \\
III & $4.66 \pm 0.39$ & $4.66 \pm 0.34$ & & $4.54 \pm 0.26$ & $4.50 \pm 0.25$ \\
V & $6.99 \pm 1.66$ & $6.93 \pm 0.43$ & & $6.88 \pm 0.42$ & $6.90 \pm 0.44$ \\
\hline
\end{tabular}

$\mathrm{R}$, right ear; L, left ear. Data were analyzed by the unpaired $t$ test. Values are mean $\pm \mathrm{SD}$.

Table 3. Comparison of interpeak latency values between groups at click intensity of $75 \mathrm{~dB} n \mathrm{HL}$

\begin{tabular}{cccccc}
\hline & \multicolumn{3}{c}{ Interpeak latency value (ms)* } \\
\cline { 2 - 3 } Interwave & $\mathrm{R}$ & $\mathrm{L}$ & & $\mathrm{c}$ Control group \\
\cline { 2 - 3 } \cline { 5 - 6 } I-III & $2.74 \pm 0.25$ & $2.81 \pm 0.22$ & & $2.70 \pm 0.24$ & $2.64 \pm 0.26$ \\
III-V & $2.33 \pm 0.24$ & $2.27 \pm 0.34$ & & $2.34 \pm 0.48$ & $2.40 \pm 0.31$ \\
I-V & $5.07 \pm 0.32$ & $5.08 \pm 0.38$ & & $5.04 \pm 0.39$ & $5.04 \pm 0.36$ \\
\hline
\end{tabular}

${ }^{*} \mathrm{R}$, right ear; $\mathrm{L}$, left ear. Data were analyzed by the unpaired $t$ test. Values are mean $\pm \mathrm{SD}$. though the sample size was small (10 infants in each group), this did not influence the validity of the statistical analysis. For example, at a power of 0.75 , the present differences in peak latencies of $\mathrm{I}$, III, and $\mathrm{V}, 0.08,0.12$, and 0.11 , respectively, in the right ear and $0.01,0.16$, and 0.03 in the left ear, with the sample size used would have been found significant at $\alpha=0.05$ only for real differences of $0.35,0.49$, and 0.62 in the right ear and $0.32,0.43$, and 0.64 in the left ear.

\section{DISCUSSION}

Auditory brainstem potentials provide an objective means for quantifying auditory system development with implications in the assessment of the effects of environmental and congenital factors during the critical period after birth $(5,6)$.

Our study has provided evidence that there is no statistically significant difference of evoked potentials in premature infants with untreated hypothyroxinemia as compared with a matched control group with normal $\mathrm{T}_{4}$ levels. The absence of any demonstrable effect of low $\mathrm{T}_{4}$ levels in our study does not exclude any harmful effect of low hormonal levels. It could be that changes in the auditory system if present, are of short duration, transient, and reversible. However, a more plausible explanation would be that untreated neonatal hypothyroxinemia has no deleterious effect on the auditory pathway. Hadeed et al. (7), who investigated the significance of transient hypothyroxinemia in premature infants, have shown that the intellectual development of infants with initially low serum $\mathrm{T}_{4}$ values was similar at $1 \mathrm{y}$ of age to a biochemically euthyroid matched control group.

We conclude that during early infancy low serum $T_{4}$ levels do not have any measurable harmful effect on auditory brainstem potentials. We believe that our study provides further evidence of the benign nature of untreated neonatal hypothyroxinemia.

Acknowledgment. The authors thank Yoav Belinski, M.A., for his cooperation in the statistical analysis of the data and Rivka Giladi for her technical assistance.

\section{REFERENCES}

1. Uhrmann S, Marks KH, Maisles MJ, Friedman Z, Murray F, Kulin HE Kaplan M, Utiger R 1978 Thyroid function in the preterm infant: a longitudinal assessment. J Pediatr 92:968-973

2. Chowdhry P, Scanlon JW, Auerbach R, Abbassi J 1984 Results of controlled double blind study of thyroid replacement in very low birthweight premature infants with hypothyroxinemia. Pediatrics 73:301-305

3. Ballard J, Novak KK, Dirver M 1979 A simplified score for the assessment of fetal maturation of newly born infants. J Pediatr 95:732-735

4. Shulman-Galambos C, Galambos R 1979 Brainstem evoked response audiometry in newborn hearing screening. Arch Otolaryngol 105:86-90

5. Starr A, Amlie RN, Martin WH, Saunders S 1977 Development of auditory function in newborn infants revealed by auditory brainstem potentials. Pediatrics 60:831-839

6. Marshall RE, Reichert TJ, Kerley SM, Davis H 1980 Auditory function in neonatal intensive care unit patients revealed by auditory brainstem potentials. J Pediatr 96:731-734

7. Hadeed AJ, Asay LD, Klein AH, Fisher DA 1981 Significance of transient postnatal hypothyroxinemia in premature infants with and without respiratory distress syndrome. Pediatrics 68:494-498 